\title{
MULTIPLEXING VOIP PACKETS OVER INTERNET TELEPHONY TRANSPORT PROTOCOL (ITTP)
}

\author{
Mosleh M. Abualhaj ${ }^{1}$, Sumaya N. Al-khatib ${ }^{2}$ and Mahmoud Baklizi ${ }^{3}$ \\ ${ }^{1}$ Department of Networks and Information Security, \\ Al-Ahliyya Amman University, Amman, Jordan \\ ${ }^{2}$ Department of Software Engineering, \\ Al-Ahliyya Amman University, Amman, Jordan \\ ${ }^{3}$ Department of Computer Networks Systems, \\ The World Islamic Sciences \& Education University W.I.S.E, Amman, Jordan
}

\begin{abstract}
Voice over IP (VoIP) is a technology of making phone calls over IP network. A considerable number of VoIP applications have emerged in the last decade, to make calls over the Internet. Accordingly, a huge number of VoIP packets are running over the Internet, which consume a considerable share of bandwidth. VoIP applications produce packets with small payload, which cause a considerable header overhead. Whereas, the header overhead is between around 46.4\% to $72.2 \%$, depends of the payload size, when using Internet Telephony Transport Protocol (ITTP)/IP protocol. VoIP packets header multiplexing is one of the most common techniques that used to reduce header overhead. In this paper, we propose a new multiplexing technique, called Delta-Multiplexing over ITTP (D-Mux-ITTP). The D-Mux-ITTP is aiming to reduce the wasted bandwidth resulting from the 26-byte ITTP/IP VoIP packet header, by combining the packets destined to the same destination into a single ITTP/IP header.
\end{abstract}

\section{KEYWORDS}

Packets Multiplexing; Bandwidth Utilization; VoIP; D-Mux-ITTP; ITTP.

\section{INTRODUCTION}

Voice over IP (VoIP) applications have propagated widely in the last decade [1]. In 2017, the size of VoIP packets, running over the internet, are expected to exceed 158 petabytes per month [2]. This noticeable propagation is driven by the fact that the VoIP calls are very cheap or free. In addition, the VoIP calls can be made from anywhere using PC, mobile, tab, and ...etc [3,4]. Notwithstanding the foregoing, VoIP still provide calls with less quality than the traditional Public Switched Telephone Network (PSTN). In addition, VoIP waste network bandwidth due to the big packet header size. Whereas, a normal VoIP packet made up of a 40-byte RTP/UDP/IP header, while, the normal VoIP packet payload size is between 10 bytes and 30 bytes [5,6]. To consider this issue, a new protocol called, Internet Telephony Transport Protocol (ITTP), was created to carry VoIP calls. The 6-byte ITTP replaces the 20-byte RTP/UDP header $[7,8]$. However, the 26-byte ITTP/IP is still considerable header in comparison to the 10 to 30 bytes payload. In this paper, we propose a new VoIP packets multiplexing technique to reduce the wasted bandwidth resulting from the 26-byte ITTP/IP VoIP packet header. In this paper, we

Natarajan Meghanathan et al. (Eds) : NLP, JSE, CST, SIP, ARIA - 2018

pp. 75-81, 2018. (C) CS \& IT-CSCP 2018

DOI : $10.5121 /$ csit.2018.80207 
propose a new VoIP packets multiplexing technique to reduce the wasted bandwidth resulting from the 26-byte ITTP/IP VoIP packet header.

The rest of this paper is organized as follows: Section 2 discusses the current multiplexing methods of VoIP packets. Section 3 demonstrates the proposed multiplexing method. Section 4 provides a discussion of the expected bandwidth utilization of the proposed method. Finally, Section 5 elaborates the conclusions.

\section{RELATED WORKS}

VoIP calls are increasingly used over the Internet. A massive number of packets are transmitted over the Internet, which consume a considerable share of bandwidth. There is outstanding effort from researchers to save the bandwidth consumption resulting from VoIP packets. One of the most common techniques is VoIP packet multiplexing. This section discusses some of existing techniques.

In 2001, Sze et al. proposed a VoIP packets multiplexing method over RTP/UDP/IP protocols. The proposed technique combines several VoIP packets transmitted to the same destination together in a single UDP/IP header, while the original RTP header is remained in each packet. However, a compression technique was applied on the RTP header. Implementation of the proposed technique showed that combining both packets multiplexing and RTP header compression succeeded in improving bandwidth utilization, whereas, bandwidth employment improved by $72 \%$ [9].

Another multiplexing method that multiplex RTP/UDP/IP packets was proposed by Abualhaj et al. in 2010. The proposed multiplexing method called Delta-multiplexing. In Delta-multiplexing, the packets that are destined to the same destination are assembled together in one UDP/IP header, while the RTP header remains unchanged. In addition, Delta-multiplexing succeeded to compress VoIP packets payload through transmitting the difference of the successive packet payloads. Combining both packet multiplexing and payload compression have shown highly efficient bandwidth exploitation. When implemented, Delta-multiplexing method succeeded to save the bandwidth between $68 \%$ and $72 \%$, depending on the VoIP packet payload size [10].

As we can see, the previous two methods were proposed to work with RTP/UDP/IP header. In 2016, M.M.Abualhaj proposed a VoIP packets multiplexing method to work with ITTP/IP protocols. The proposed multiplexing method called ITTP-Mux. In ITTP-Mux, the packets that are destined to the same destination are assembled together in one IP header, while the ITTP header remains unchanged. Implementation of ITTP-Mux method showed high reduction in packet overhead, especially when compared with the traditional ITTP protocol (without multiplexing), which eventually save the bandwidth. The simulation result showed that bandwidth exploitation improved by up to $29.1 \%$ in the tested cases [11]

In this paper, we propose a new multiplexing method called Delta-Multiplexing over ITTP (DMux-ITTP). D-Mux-ITTP combines between both packet multiplexing as in [11] and packet payload compression as in [11]. The following section discusses the architecture of the proposed method, namely; D-Mux-ITTP method.

\section{D-MUX-ITTP ARCHITECHTURE}

This section discusses the design of the proposed method, namely; D-Mux-ITTP. The D-MuxITTP consists of two components. First is the Sender-Delta-Multiplexing (SDM) component, which reside at the sender side. Second is the Receiver-Delta-DeMultiplexing (RDD) component, which reside at the receiver side. The SDM component works as follows: i) it extract the payload 
of the packets transmitted to the same destination, ii) the extracted payloads are subtracted from each other, which produces a smaller payload called s-payload iii) the SDM attaches a miniheader to each s-payload, to distinguish these payloads, which produces a small packet called mini-packet, and iv) these mini-packets are combined together in one ITTP/IP packet. Figure 1 demonstrates the SDM component process.

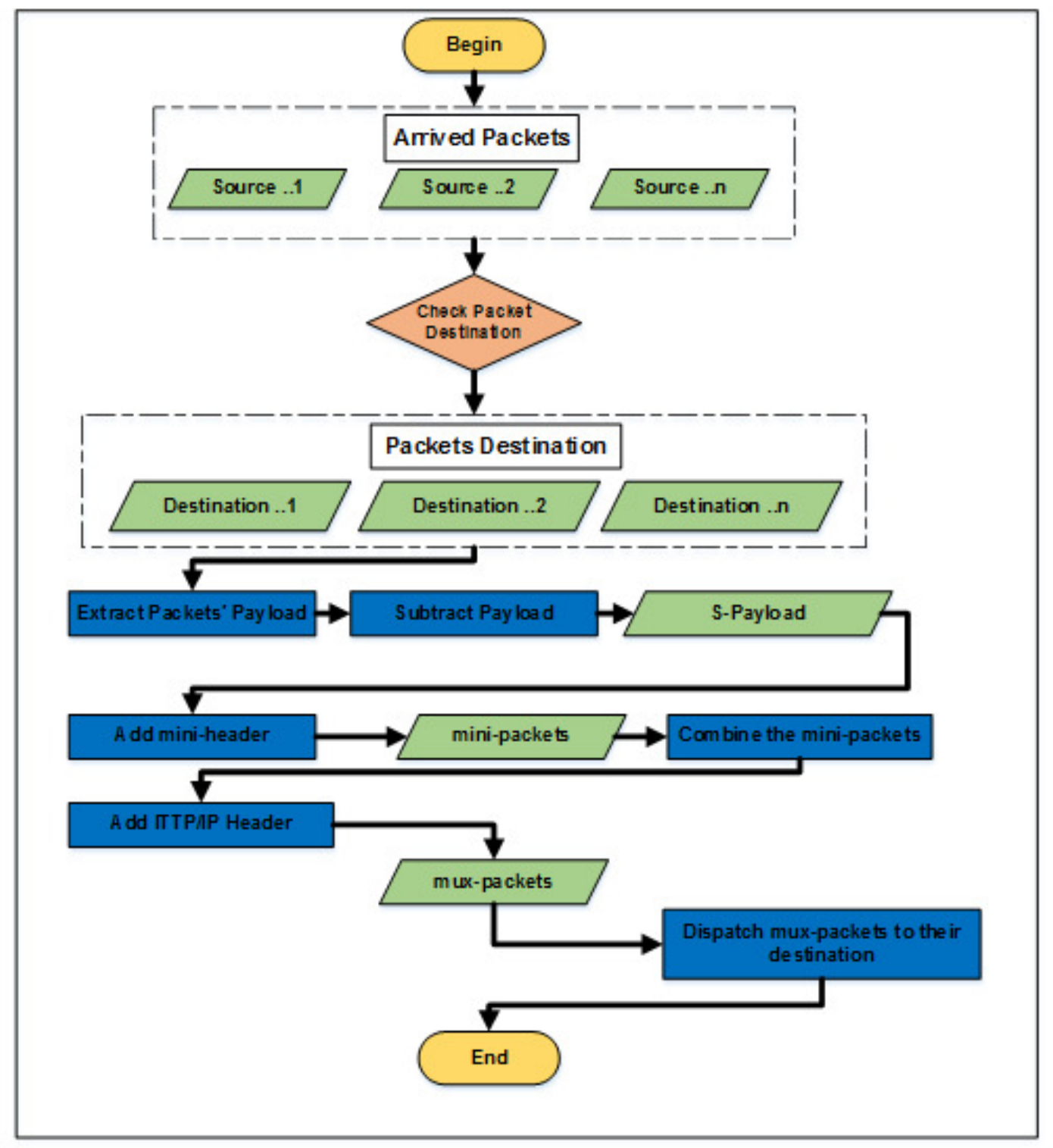

Figure 1. SDM Component Process

The RDD component works as follows: i) it separates the received ITTP/IP packet into minipackets by inspecting the mini-header ii) it then removes the mini-header from the mini-packet iii) after that it restores the original size of the payload, iv) and finally, the RDM attach the ITTP/IP packet to the restored payload, which re-construct the original packet. Figure 2 demonstrates the RDD component process. Figure 3 demonstrates the D-Mux-ITTP method architecture. 


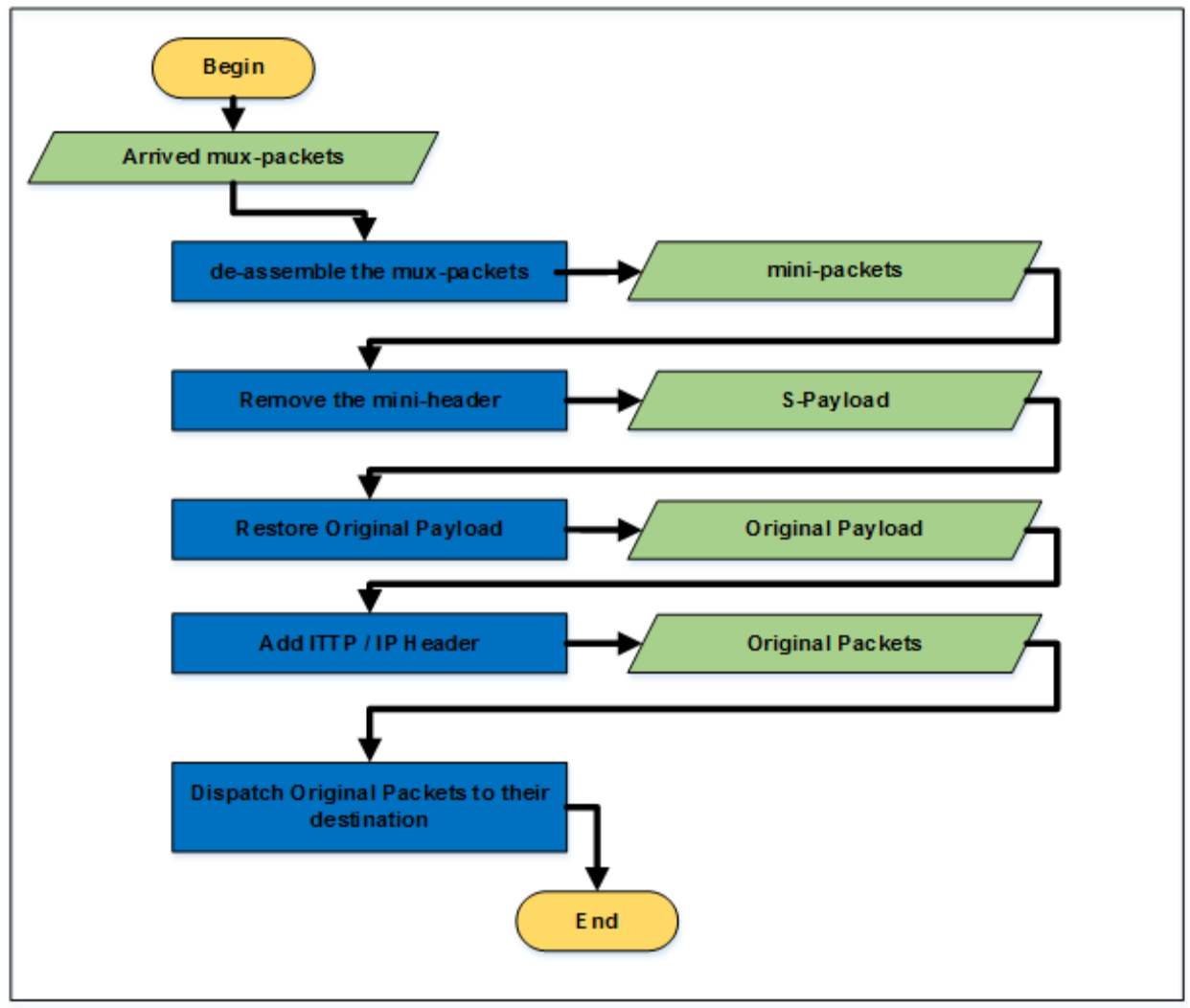

Figure 2. RDD Component Process

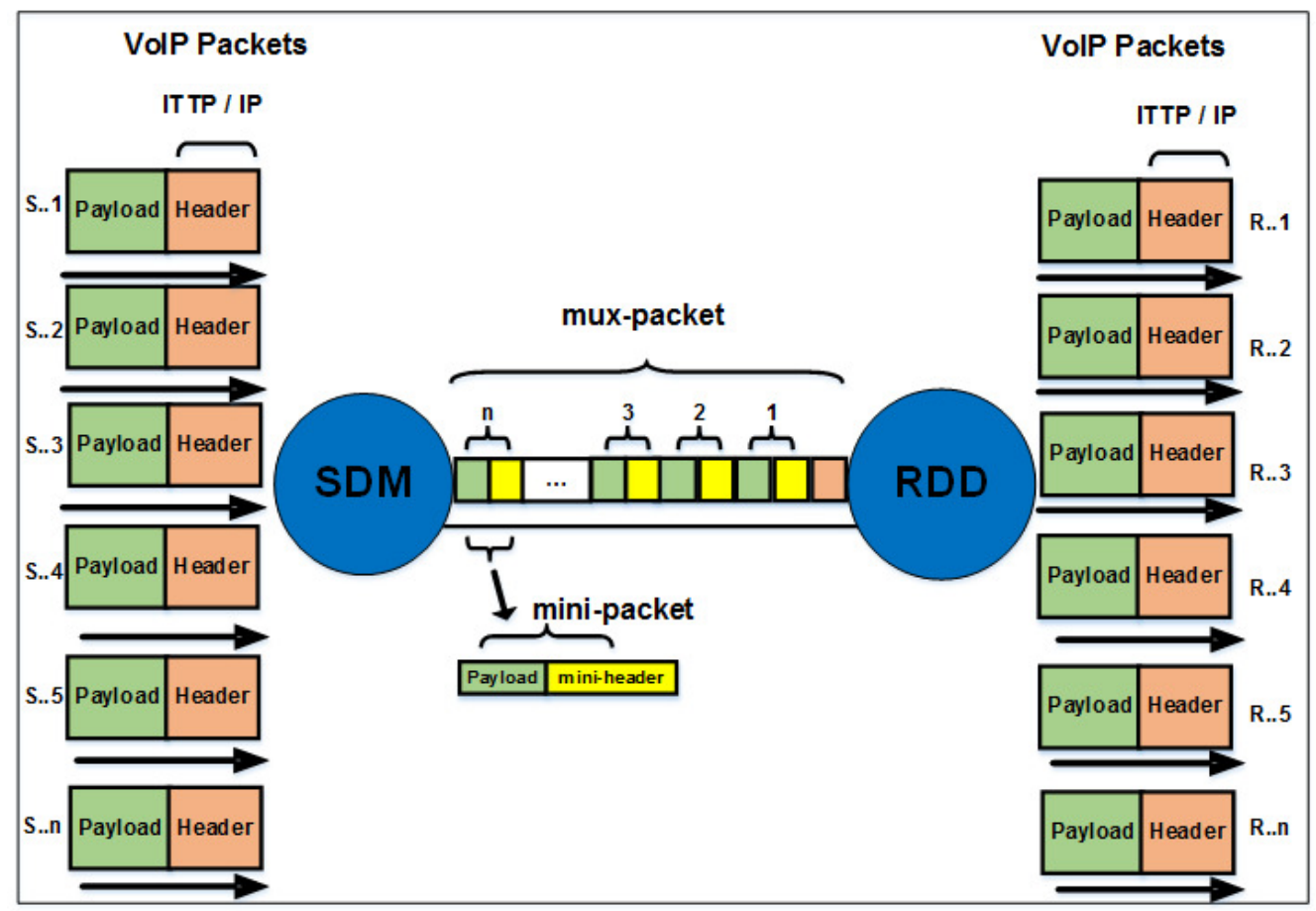

Figure 3. D-Mux-ITTP Method Architecture 
The D-Mux-ITTP achieves bandwidth utilization through two phase. In phase1, several VoIP packets from different sources are combined together in one ITTP/IP header. This phase reduces the header overhead resulting from attaching a separate header to each packet. In phase2, the VoIP packets payload, before get combined together, are assumed to be integer number and subtracted from each other. This phase reduces the payload size through transmit the difference between the payloads instead of the full payloads size.

\section{D-MUX-ITTP BRANDWIDTH UTILIZATION EFFICIENCY}

This section discusses the D-Mux-ITTP bandwidth utilization efficiency in comparison to the traditional method (no multiplexing). The traditional VoIP packet payload size between 10 and 30 bytes $[5,6]$. Accordingly, the header overhead resulting from the 26-bytes ITTP/IP header, which is the relative ratio between the header size and the packet size, is between around $46.4 \%$ to $72.2 \%$. Figure 4 demonstrates the header overhead ratio with different payload sizes.

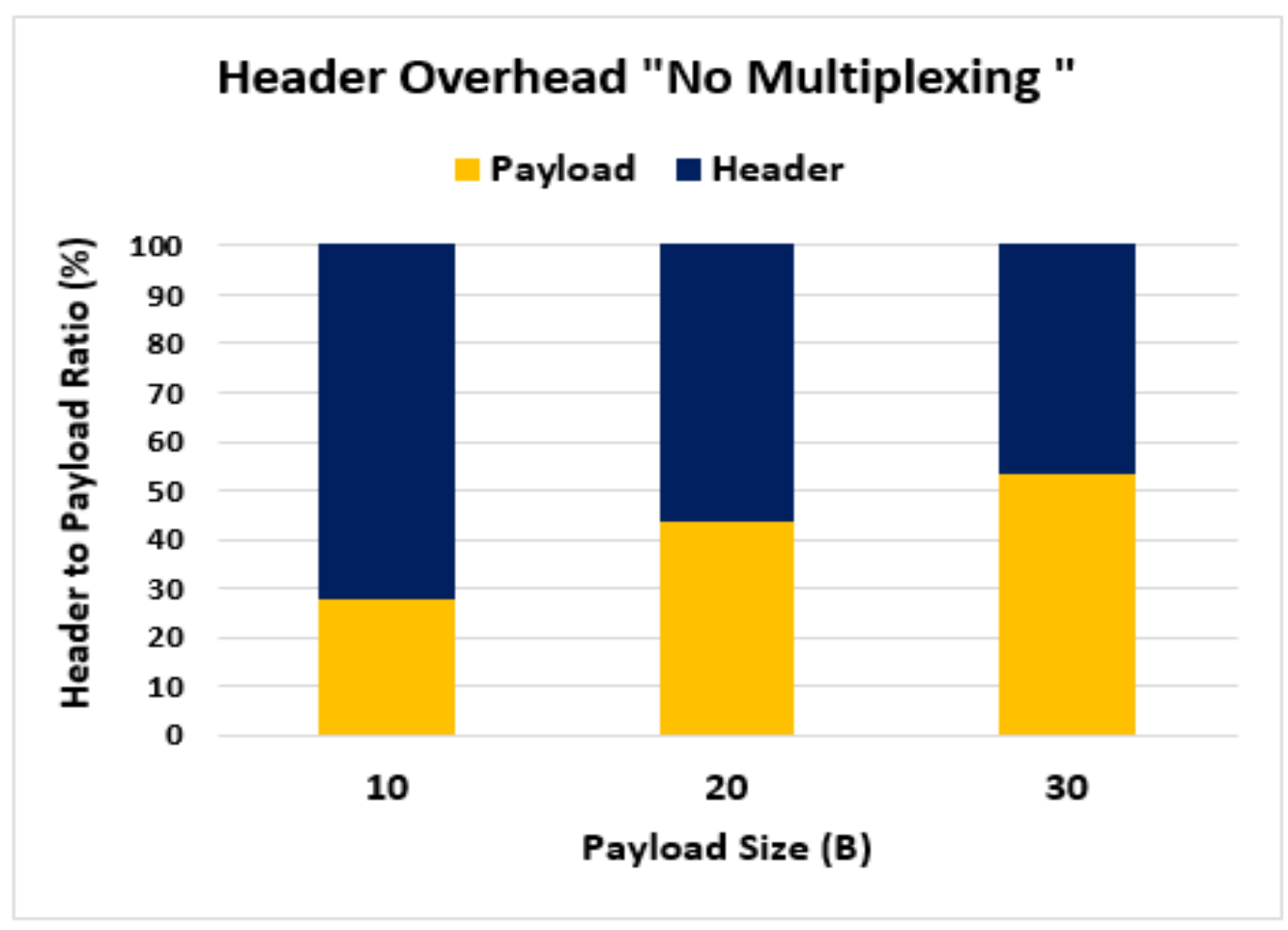

Figure 4. Header Overhead Ratio (No Multiplexing)

The aim of the D-Mux-ITTP method is to reduce the wasted bandwidth resulting from the considerable ITTP/IP header overhead. D-Mux-ITTP method accomplish this by multiplexing several VoIP packets payload in a single ITTP/IP header, which reduces the ITTP/IP header overhead. This is efficiently improve bandwidth utilization, depending on the number of multiplexed packets. Figure 5 demonstrates the header overhead ratio when multiplexing several packets in single ITTP/IP header, assuming packet's payload size is 20-byte. Note that Figure is only for demonstration purpose and the numbers are approximated, based on the relative ratio between the header size and the multiplexed packets size. In future work, we will implement the D-Mux-ITTP method and provide an accurate values of the bandwidth utilization efficiency when using it. 


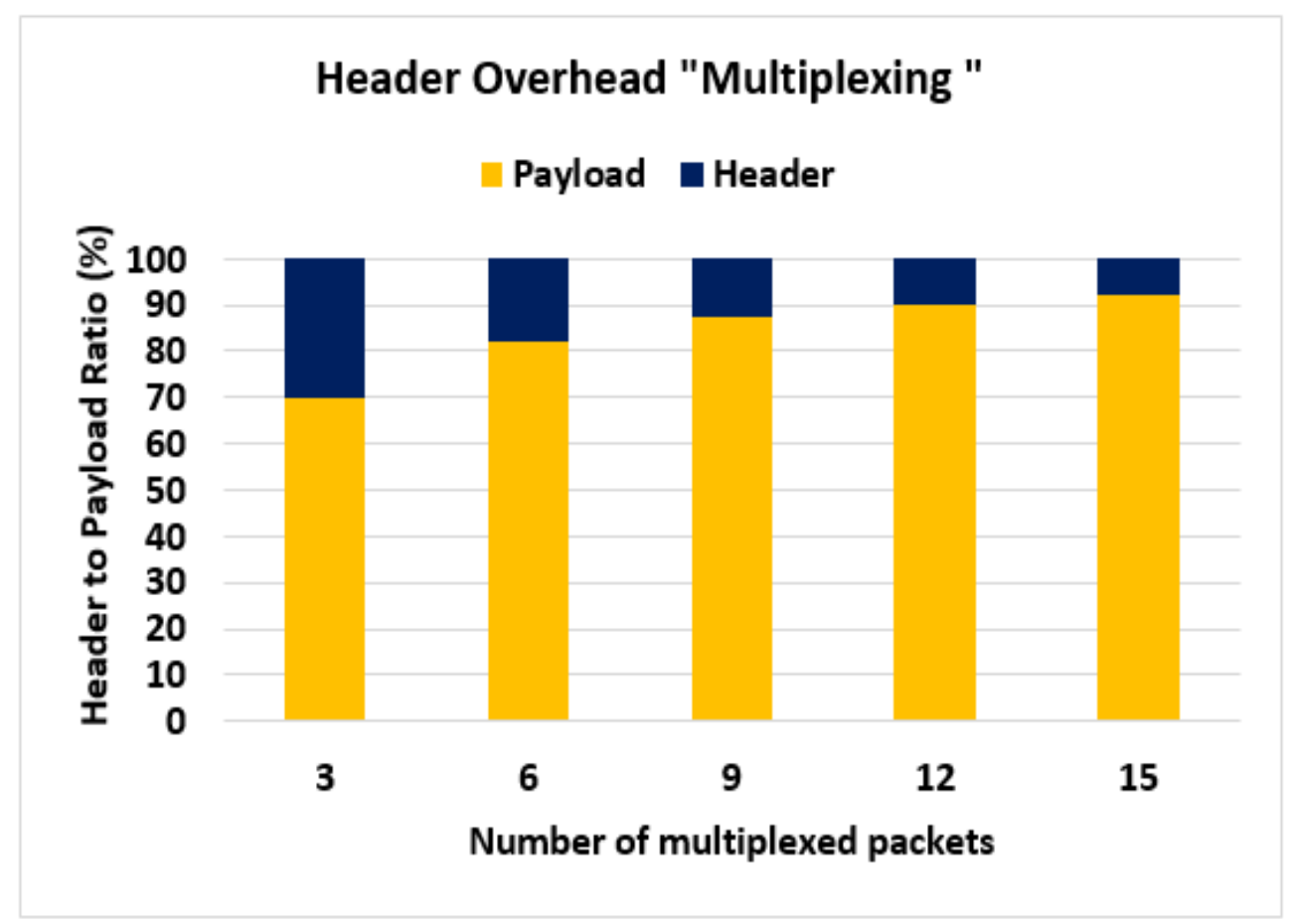

Figure 5. Header Overhead Ratio (Multiplexing)

\section{CONCLUSIONS}

In this paper, we proposed a new multiplexing method called D-Mux-ITTP. The aim of the DMux-ITTP method is to efficiently improve bandwidth exploitation, by reducing the wasted bandwidth resulting from the considerable ITTP/IP header overhead. The D-Mux-ITTP achieves this by i) combining several VoIP packets payloads in a single ITTP/IP header and ii) by transmit the difference between the payloads instead of the full payloads size. The D-Mux-ITTP consists of SDM and RDD entities. SDM performs packets multiplexing and the sender side, while, RDD performs packets de-multiplexing and the receiver side. Future work will implement the D-MuxITTP method and provide a detailed discussion and analysis of the D-Mux-ITTP bandwidth utilization, in terms of header overhead, saved bandwidth, call capacity, and goodput. In addition, the impact of the D-Mux-ITTP on the network performance and call quality will be provided. Network performance and call quality will be measured through delay, overload, congestion, and ...etc.

\section{REFERENCES}

[1] MM Abualhaj, M Kolhar, K Qaddoum, AA Abu-Shareha, (2016) "Multiplexing VoIP Packets over Wireless Mesh Networks: A Survey, KSII Transactions on Internet \& Information Systems”, Vol. 10, No.8.

[2] http://www.statista.com/statistics/267183/forecast-for-the-worldwide-voip-traffic/, November-2017.

[3] AbuAlhaj, Mosleh, Manjur S. Kolhar, M. Halaiyqah, O. Abouabdalla and R. Sureswaran, (2009) "Multiplexing SIP applications voice packets between SWVG gateways", in Proc. of International Conference on Computer Engineering and Applications (ICCEA 2009). 
[4] Manjur, S. K., Mosleh Abu-Alhaj, Omar Abouabdalla, Tat-Chee Wan, Rahmat Budiarto and Ahmed M. Manasrah, (2011), "Conference gateway for heterogeneous clients: Real time switching clients and interasterisk exchange clients", International Journal of Innovative Computing Information and Control 7, No. 1, 395-406.

[5] MM Abu-Alhaj, A Manasrah, M Baklizi, N Abdullah, (2011) "Transport layer protocols taxonomy from Voice over IP perspective”, Advanced Computing: An International Journal ( ACIJ ), Vol.2, No.4.

[6] M Abu-Alhaj, MS Kolhar, M Halaiyqah, O Abouabdalla, (2009) “ MuxComp-A New Architecture to Improve VoIP Bandwidth Utilization”, International Conference on Future Networks.

[7] Abu-Alhaj, Mosleh M., S. K. Manjur, R. Sureswaran, Tat-Chee Wan, Imad J. Mohamad and Ahmed M. Manasrah, (2012) "ITTP: A New Transport Protocol for VoIP Applications", International Journal of Innovative Computing, Information and Control (IJICIC), Vol. 8, No. 3(A), pp. 1879-1895.

[8] A Kayed, MM Abualhaj, M Alharibat, (2013),"PERFORMANCE COMPARISON OF IAX AND ITTP VoIP PROTOCOLS”, International Journal of Academic Research, Vol. 5, No. 2.

[9] Sze, H. P., Soung C. Liew, Jack YB Lee and Danny Yip, (2002), “A multiplexing scheme for H. 323 voice-over-IP applications”, Selected Areas in Communications, IEEE Journal, No. 7, pp. 1360-1368.

[10] Abu-Alhaj, Mosleh M., (2015)," ITTP-MUX:An efficient multiplexing mechanism to improve voip applications bandwidth utilization", International Journal of Innovative Computing, Information and Control (IJICIC), Vol. 11, No. 6, pp. 1-1503-0010.

[11] Abu-Alhaj, Mosleh M., Manjur S. Kolhar, Lingeswari V. Chandra, O. Abouabdalla and Ahmed M. Manasrah, (2010), "Delta-Multiplexing: A Novel Technique to Improve VoIP”. 